\title{
25 Research Square \\ Dental pulp stem cells as a therapy for congenital entero-neuropathy
}

\section{Shunichi Kajioka}

Kyushu University

Junko Yoshizumi

Kyushu University

Yoshinao Oda

Kyushu University

Naoko Iwata

Nagoya University

Chiho Takai

Nagoya University

Shinsuke Nakayama ( $\nabla$ snak@med.nagoya-u.ac.jp )

Nagoya University

Koichiro Yoshimaru

Kyushu University

Takayoshi Yamaza

Kyushu University

Soichiro Sonoda

Kyushu University

\section{Yusuke Yanagi}

Kyushu University

\section{Tomoaki Taguchi}

Kyushu University

Toshiharu Matsuura

Kyushu University

\section{Research Article}

Keywords: Gastrointestinal motility, pulp stem cells, deciduous tooth, Ednrb gene

Posted Date: July 21st, 2021

DOI: https://doi.org/10.21203/rs.3.rs-596893/v3 
License: (c) (i) This work is licensed under a Creative Commons Attribution 4.0 International License. Read Full License 


\section{Abstract}

Hirschsprung's disease (HSCR) and its allied disorders are congenital entero-neuropathies with life-long implications in many cases. Here we report the effects of intravenous transplantation of cultured dental pulp stem cells derived from deciduous teeth (dDPSCs) into 'Japanese fancy-1' (JF1) mice with enteroneuropathy caused by Ednrb mutation. Intravenously injected dDPSCs (multipotent neural crest cells with low immunogenicity) migrated to affected regions of the intestine through interactions between stromal cell-derived factor-1 $\mathrm{a}$ and C-X-C chemokine receptor type-4. Notably, transplanted dDPSCs differentiated into both enteric neurons and pacemaker interstitial cells to correct abnormalities in the electrical and mechanical activities of the proximal colon. dDPSC transplantation also led to repair of the small intestinal mucosa, changes in the gastrointestinal microbiota, improvements in nutritional status and prolongation of survival. We anticipate that dDPSC transplantation could be developed into a novel cellbased therapy for HSCR and its allied disorders.

\section{Introduction}

Hirschsprung's disease (HSCR) and its allied disorders are congenital entero-neuropathies that occur with an incidence of $\sim 1$ per 5000 livebirths in Japan (1-3). HSCR is a potentially fatal disorder with clinical features that include constipation, abdominal distension, megacolon and intestinal obstruction (1-3). The current management of HSCR involves surgery to remove or bypass affected segments of the bowel (4). Nevertheless, many children suffer from life-long complications due to the dysfunction of 'healthy' bowel retained during surgery (5) and the consequences of additional palliative surgery such as proctectomy and enterostomy. Therefore, novel therapies are urgently needed to improve outcomes and quality of life in patients with HSCR.

HSCR was first reported in 1888 by Hirschsprung, who described two infants with local defects of enteric neurons (6). The pathogenesis of HSCR is incompletely understood but thought to involve the failure of enteric neural crest-derived cells (NCCs) to complete their colonization of the distal intestine during foetal development. Since the initial discovery that enteric neural circuits play an essential role in peristaltic movements of the gut (the 'law of the intestine' proposed by Bayliss and Starling in 1899) (7), it has become established that intestinal motility is coordinated by an intrinsic nervous system. In the 1990s, the interstitial cells of Cajal (ICCs) were identified as specialized cells that act as an intestinal pacemaker (8). ICCs (especially those in the myenteric plexus) are thought to contribute to the spatial organization of gut motility because of their network-like structure. Cooperation between the enteric nervous system (ENS) and ICC 'pacemaker' system is essential for the coordination of complex intestinal movements $(9,10)$, and this cooperation between the ENS and ICCs is thought to be impaired in HSCR and its allied disorders. The complex interaction between the ENS and ICCs may explain why familial RET mutations, which are known to affect colonization of the intestine by NCCs, exhibit incomplete penetrance (11).

Dental pulp stem cells derived from deciduous teeth (dDPSCs) are cranial neural crest-derived mesenchymal stem cells (MSCs) that can differentiate to form structures such as the pulp-dentin 
complex due to their multilineage potential (12-14). Our cultured dDPSCs are multipotent cells that express NCC markers and have low levels of class II HLA, which is important for immune tolerance (15). In view of their characteristics, we hypothesized that dDPSCs may have potential for development into a novel cell-based therapy for entero-neuropathies. Here we report the effects of intravenous transplantation of dDPSCs into the Japanese Fancy-1 (JF1) mouse, which is an established animal model of entero-neuropathy. The JF1 mouse carries a homozygous mutation at the piebald locus $\left(E d n r b^{s}\right)$ that encodes the endothelin-B receptor (ETBR), and the phenotype is characterized by a sparse network of myenteric ganglia, especially in the proximal colon (16-19). We show that intravenously injected dDPSCs migrate to affected regions of the intestine through interactions between stromal cellderived factor-1a (SDF1a) and C-X-C chemokine receptor type-4 (CXCR4). Furthermore, the transplanted dDPSCs differentiate into both pacemaker cells and enteric neurons in the proximal colon to restore the slow rhythmic potentials observed in wild-type mice. Our findings imply that congenital enteroneuropathies involve defects of the intestinal pacemaker system and that dDPSCs have potential for development into a new treatment for HSCR in humans.

in the gastrointestinal microbiota, improvements in nutritional status and a prolongation of survival. We anticipate that dDPSC transplantation could be developed into a novel cell-based therapy for HSCR and its allied disorders.

\section{Results}

\section{dDPSCs migrate to the intestine}

JF1 mice had a shorter body length than age-matched wild-type B6 mice, and their colons exhibited faecal stasis and reduced mucosal thickness (Extended Data Fig. 1a-c). Proximal colon levels of endothelin receptor type $\mathrm{B}(E d n r b) \mathrm{mRNA}$ and protein were lower in JF1 mice than in B6 mice (Extended Data Fig. 1d-f). Compared with B6 mouse colon, JF1 mouse colon had higher levels of macrophage infiltration, intestinal endotoxin, interleukin-2 (IL-2) and macrophage inflammatory protein-2 (MIP2) (Fig. 1a-e), higher faecal bacterial content, a higher proportion of Bacteroidetes (but not Firmicutes or other bacteria) in the intestinal microbiota, and a lower Gini-Simpson index (Extended Data Fig. 2). Hence, JF1 mouse colon exhibits intestinal inflammation and bacterial imbalance similar to that observed in Ednrb knock-out mice ${ }^{20}$.

Stromal cell-derived factor-1a (SDF1a) is upregulated in inflamed tissue, and its receptor (CXCR4) ${ }^{21}$ is expressed in MSCs such as dDPSCs and bone marrow MSCs (BMMSCs). SDF1a-positive cells were abundant in the myenteric plexus of JF1 mouse proximal colon (Fig. 1f), raising the possibility that dDPSCs and BMMSCs might target intestinal regions exhibiting hypoganglionosis-related local inflammatory changes. Therefore, our subsequent experiments investigated the effects of intravenous injection of dDPSCs into JF1 mice at postnatal week 11 (P11W), and in some experiments we compared the effects of transplanted dDPSCs with those of intravenously injected BMMSCs or human skin fibroblasts (FBs) (Fig. 1g). Cultured dDPSCs at passage 3 (P3) expressed KIT (CD117, a receptor tyrosine 
kinase) and CXCR4 (Fig. 1h,i), retained the characteristics of neural crest cells, and had low immunogenicity (Extended Data Fig. 3).

We investigated the migration of transplanted dDPSCs (P11W) using cells labelled with 1,1'-dioctadecyl3,3,3',3'-tetramethylindotricarbocyanine iodide (DiR). In vivo fluorescence imaging of dDPSC-transplanted JF1 mice (dDPSC-JF1 mice) revealed that DiR-labelled dDPSCs were widely distributed on day 1 but had migrated to the intestine by day 7 (Extended Data Fig. 4a). Ex vivo fluorescence imaging 9 weeks after transplantation revealed a high fluorescence intensity in the intestine (stomach, distal ileum and proximal colon), spleen and liver (Fig. 1j, Extended Data Fig. 4b). Intraperitoneal administration of a neutralizing antibody to SDF1a almost completely abolished the intestinal accumulation of DIR-labelled dDPSCs (Fig. 1j, Extended Data Fig. 4a,b), suggesting that SDF1a-CXCR4 interactions play a role in dDPSC migration to the intestine. Little or no fluorescence was detected in B6 mice injected with DiR-labelled dDPSCs (Extended Data Fig. 4c), implying that dDPSCs migrate specifically to regions of hypoganglionosis. Carboxyfluorescein diacetate succinimidyl ester (CFSE)-labelled dDPSCs were observed in the proximal colon of dDPSC-JF1 mice 3 days after transplantation, whereas CFSE-labelled BMMSCs were not detected (Fig. 1k). Immunostaining for human mitochondria (hMt) identified dDPSCs in the proximal colon 12 weeks after transplantation (Fig. 1I). The above results demonstrate that SDF1a-CXCR4 interactions play a crucial role in the migration of dDPSCs to regions of hypoganglionosis in JF1 mouse intestine.

\section{dDPSCs regenerate neurons and ICCs}

Morphological investigations (Fig. 2a,b) revealed that JF1 mouse proximal colon exhibited poorer development of the intestinal musculature and myenteric region (haematoxylin-eosin staining), fewer myenteric ganglion cells (stained with the neuron-specific marker, HuC/HuD), and weaker immunostaining for neurofilament M (NFM, a peripheral ganglion marker) and KIT (a pacemaker interstitial cell marker). These observations suggest that congenital entero-neuropathy is accompanied by poor development of the pacemaker system ${ }^{22,23}$. Notably, dDPSC-JF1 mouse colon exhibited increased numbers of enteric neurons and pacemaker interstitial cells (Fig. 2a,b). Immunohistochemistry experiments in dDPSC-JF1 mice revealed that hMt was co-expressed in some HuC/HuD-positive and NFM-positive neurons in the myenteric plexus region (Fig. 2c,d) and in numerous KIT-positive cells (Fig. $2 e)$, indicating that myenteric neurons and pacemaker cells had developed from dDPSCs.

Since inadequate interactions between trophic factors may contribute to hypoganglionosis ${ }^{1-4}$, we assessed the effects of dDPSCs on trophic factors. The concentrations of mouse nerve growth factor (NGF), glial cell-derived neurotrophic factor (GDNF) and stem cell factor (SCF, a KIT ligand) were similar between B6 and JF1 mice (Fig. 2f). dDPSC transplantation increased the protein levels of mouse NGF and GDNF and human GDNF, NGF and SCF (Fig. 2f). These findings are consistent with dDPSC-mediated paracrine actions. 
We measured the mechanical and electrical activities of the second part of the proximal colon (Extended Data Fig. 1b) to assess the functional effects of dDPSC transplantation. Contractile responses to acetylcholine (Ach), endothelin-1 (ET-1) and electrical field stimulation (EFS) were smaller in JF1 mice than in B6 mice but comparable between dDPSC-JF1 mice and B6 mice (Extended Data Fig. 5a-d). The relaxation response to a nitric oxide (NO) donor was smaller in JF1 and dDPSC-JF1 mice than in B6 mice (Extended Data Fig. 5e). Contractile responses to a high concentration $(60 \mathrm{mM})$ of extracellular $\mathrm{K}^{+}$were similar among the three groups (Extended Data Fig. $5 f$ ), suggesting that the contractile ability of colonic smooth muscle was preserved in JF1 mice. The above findings agree well with the data in Fig. 2 (recovery of myenteric neurons after dDPSC transplantation).

We also evaluated the characteristics of spontaneous colonic contraction. The second part of the proximal colon exhibited irregular, high-frequency, small-amplitude, transient contractions in JF1 mice, which contrasted with the prolonged phasic contractions occurring at intervals of several minutes in B6 mice (Fig. 3a-c). dDPSC transplantation into JF1 mice restored the magnitude and frequency of spontaneous contractions to levels comparable to those in B6 mice (Fig. 3a-c). dDPSC transplantation also restored spontaneous contraction magnitude in the first part of the proximal colon (Extended Data Fig. $5 \mathrm{~g})$.

Spatiotemporally-coordinated electrical activity underlies the complex movements of the gastrointestinal tract $^{25}$. We used the dialysis membrane-reinforced microelectrode array (MEA) technique to monitor field potentials in muscle sheets isolated from the second part of the proximal colon (Extended Data Fig. 6ac). Samples from B6 mice displayed basal electrical oscillations with a period of $3.78 \pm 0.22 \mathrm{~s}(n=10$ recording regions, $N=6$ animals) in most of the recording area, and occasionally a large potential occurred (Fig. 3d, Extended Data Fig. 6d). Basal rhythmic oscillations rarely occurred in JF1 mouse colon (observed in $1 / 10$ recording regions, $N=3$ ), whereas electrical complexes of rapid and slow potentials occurred frequently (Fig. 3e, Extended Data Fig. 6e). The rapid potentials propagated in limited microregions (suggesting poor spatial synchronicity) with a frequency $(1-2 \mathrm{~Hz})$ comparable to that of myoelectric complexes ${ }^{26-28}$. dDPSC transplantation into JF1 mice restored the basal rhythmic oscillations, which occurred with an interval $(4.12 \pm 0.20 \mathrm{~s}, n=10, N=3)$ comparable to that in B6 mice (Fig. 3f, Extended Data Fig. 6f). However, the basal oscillation phase varied within the small recording area, and rapid potentials occurred locally in some samples, suggesting incomplete recovery of spatiallycoordinated motor function. Spectral analysis $(0.1-15 \mathrm{~Hz})$ revealed that the electrical potential magnitude in JF1 mice was smaller than that in B6 mice but restored by dDPSC transplantation (Fig. 3g). The low-frequency component was smaller in JF1 mice than in B6 mice but comparable between dDPSCJF1 and B6 mice (Fig. 3h,i), indicating that colons from dDPSC-JF1 and B6 mice exhibited similar regular basal rhythmicity. The effects of dDPSC transplantation on electrical characteristics are consistent with the restoration of myenteric neurons and pacemaker interstitial cells (Fig. 2).

\section{dDPSCs improve nutrition and survival}


JF1 mice exhibited slower bodyweight gain (from P11W to P35W) than B6 mice, and intravenous transplantation of dDPSCs (but not BMMSCs or FBs) caused a small but significant enhancement of bodyweight gain (Fig. 4a, Extended Data Fig. 1a). Intraperitoneal injection of dDPSCs did not improve bodyweight gain in JF1 mice (data not shown). Median survival time (up to P90W) of JF1 mice was shorter than that of B6 mice (77.8 weeks vs. 112.2 weeks, $n=10, P=0.004$ ) and prolonged by the transplantation of dDPSCs ( 90.2 weeks, $n=10, P=0.039$ vs. JF1 mice) but not BMMSCs ( 80.0 weeks, $n=$ $10, P=0.492$ vs. JF1 mice) or FBs (73.8 weeks, $n=10, P=0.481$ vs. JF1 mice; Fig. 4b). dDPSC transplantation into JF1 mice improved colonic transportation (ex vivo imaging of colonic content after a 24-h fast; Fig. 4c), restored long bone (tibial) mineral density (micro-computed tomography; Fig. 4d,e), enhanced hepatic glucose storage and improved the small intestinal mucosa (Extended Data Fig. 1c, Extended Data Fig. 7). These results indicate that multipotent dDPSCs can partially correct enteroneuropathy in JF1 mice (Fig. 4f).

Finally, we investigated potential off-target effects of dDPSC transplantation. dDPSC-JF1 mice exhibited no morphological alterations or T-cell accumulation in the lungs, liver or kidneys and no changes in the serum levels of enzymes related to the function of these organs or cytokines related to systemic inflammation (Extended Data Fig. 8).

\section{Discussion}

Experiments in animal models of spinal cord transection and autoimmune encephalitis suggest that dDPSCs can differentiate into neural crest-derived cells (neurons and glial cells) 29,30 . The treatment of congenital entero-neuropathies is more challenging because the complex cytoarchitecture of the gastrointestinal tract develops from three germ layers. Our study shows that dDPSCs have potential for development into a therapy for entero-neuropathies due to their pluripotent characteristics. Intravenous administration of dDPSCs improved the survival and nutritional status (bodyweight and glucose uptake) of JF1 mice. We speculate that the latter effect was partly due to repair of the mucosa (endoderm) by epithelial cells generated by dDPSCs. Furthermore, staining for hMt indicated that dDPSCs differentiated into pacemaker interstitial cells (mesoderm) and enteric neurons (ectoderm). The ability of dDPSCs to undergo multi-lineage differentiation is critical for their therapeutic potential because numerous cells and mechanisms underlie coordinated gut motility ${ }^{10}$ and because congenital entero-neuropathies likely involve not only enteric neuron loss but also other impairments, as seen in JF1 mice. Additionally, our data suggest that paracrine mechanisms contribute to the therapeutic effects of dDPSC transplantation.

According to our observation, dDPSCs may have advantages over BMMSCs, FBs and other stem cell types (Supplementary Discussion 1) in the treatment of refractory bowel disorders. Undifferentiated MSCs such as dDPSCs express high levels of inflammation-activated adhesion proteins that enables them to avoid mechanical trapping in healthy capillaries and settle in regions affected by inflammation ${ }^{31}$. The lack of intestinal accumulation of dDPSCs in B6 and anti-SDF1a antibody-treated JF1 mice demonstrates the crucial role of SDF1a-CXCR4 interactions in the migration of dDPSCs to affected bowel regions. However, CXCR4-expressing BMMSCs did not colonize the myenteric region or improve the 
nutritional status of JF1 mice, indicating that mechanisms other than SDF1a-CXCR4 interactions contribute to the migration and colonization processes. For example, dDPSCs express KIT whereas BMMSCs do not, and it has been reported that SCF (a KIT ligand released from enteric neurons and smooth muscle cells) induces the differentiation of MSC-derived progenitor cells into ICCs ${ }^{24}$. It is likely that transplanted dDPSCs differentiate into progenitors of enteric neurons and pacemaker cells, which act synergistically to correct bowel dysmotility through interactions between SCF and KIT and the release of neurotransmitters and hormones that promote the development of ICCs ${ }^{24}$.

Various cell types have been evaluated for possible use as a cell-based therapy for HSCR and its allied disorders. Enteric NCC-derived progenitors from mice successfully develop into functional neurons when grafted into the colon of wild-type recipient mice with the same background ${ }^{32}$. Moreover, pluripotent stem cells such as embryonic stem cells (ESCs) and induced pluripotent stem cells (iPSCs) can regenerate gutlike organoids that exhibit neural responses and pacemaker potentials ${ }^{33,34}$. Recently, an ESC-derived NCC lineage was shown to engraft successfully and reduce disease-related mortality in an animal model of HSCR (mice with an Ednrb mutation) ${ }^{35}$. As with dDPSCs, the therapeutic effect of NCCs derived from pluripotent stem cells may be enhanced by the simultaneous development of pacemaker cell lineages. However, the clinical application of ESC- and iPSC-derived lineages is currently controversial due to possible carcinogenic and immunogenic effects ${ }^{36,37}$, which highlights the low immunogenicity of dDPSCs as an important advantage (Extended Data Fig. 3). We observed hMt-containing neurons and pacemaker cells in the colon of JF1 mice 12 weeks after transplantation as well as an improvement in both mechanical and electrical function (Fig. 2 and 3). Moreover, the high expression of phosphatase and tensin homolog (PTEN) is thought to reduce the carcinogenicity of dental pulp stem cells ${ }^{38}$. Although autologous transplantation would be preferable for the clinical application of dDPSCs, the low immunogenicity of these cells would likely allow allogeneic transplantation of dDPSCs obtained from deciduous or extracted teeth.

The colon exhibits characteristic patterns of electrical potentials. Slowly oscillating potentials (occurring at intervals of 3-4 s) are prominent in the proximal colon of wild-type mice. KIT-positive pacemaker cells are the likely origin of slow potentials ${ }^{22,23,39}$. Spontaneous myoelectric complexes comprising rapid and slow components occur in the mid-to-distal colon, and this electrical activity is considered neurogenic in origin and the basis of propulsion ${ }^{28}$. Activity resembling myoelectric complexes is observed in JF1 mouse proximal colon despite the poor spatial synchronicity, suggesting that loss of spontaneous rhythmicity generated by network-forming pacemaker cells is compensated for by the relatively preserved enteric neurons. We propose that both the ENS and network-forming pacemaker system are impaired in enteroneuropathies. This hypothesis would account for some of the observed discrepancies in colonic motility, such as bi-directional propagation of colonic migrating motor complexes ${ }^{27}$.

Heterozygous and homozygous $s /$ mutations of Ednrb cause similar reductions in the myenteric ganglia of mice, but the homozygous $s /$ mutation is lethal due to a complete absence of motility ${ }^{40}$, which would suggest a role for factors other than ENS impairment. Mutations of Ret are known to be associated with 
defects in enteric neurons, and homozygous mutations of Ret also yield HSCR-like mice, whereas the heterozygous mutation is asymptomatic. Recent studies of the Ret-null mouse embryo suggest that Ret has much broader effects than enteric neurogenesis ${ }^{41}$, as we suggest for the Ednrb mutation. We speculate that symptoms of bowel dysmotility in animal models of congenital entero-neuropathies arise from the additional impairment of the pacemaker system, because coordinated gastrointestinal movement is maintained by a multitude of cooperating systems. This may also be true in humans, particularly given the discrepancy between bowel motility symptoms and morphological defects in the ENS.

In conclusion, the pacemaker and intrinsic nervous systems of the proximal colon are impaired in JF1 mice with Ednrb mutation. Therefore, appropriate cell-based therapy requires cells with multi-lineage potential. Notably, transplanted dDPSCs migrate to affected regions of the colon and improve the electrical and mechanical activity, likely because they differentiate into pacemaker interstitial cells and enteric neurons. Further investigations are required to optimise the transplantation technique, for example by improving cell migration to affected intestinal regions and promoting cell differentiation to repair defective tissues. Nevertheless, we consider dDPSC transplantation as a promising cell-based therapy for congenital entero-neuropathies, especially for patients with pacemaker dysfunction in apparently 'healthy' intestinal segments.

\section{Methods}

\section{Ethics statement}

Healthy human deciduous teeth were collected as discarded biological/clinical samples from three healthy donors (5-7 years old) who attended the Department of Pediatric Dentistry of Kyushu University Hospital. The parents/guardians of the donors provided written informed consent that stem cells harvested from the teeth could be used for research into regenerative medicine for congenital diseases. Human peripheral blood mononuclear cells (PBMNCs) were collected from peripheral venous blood obtained from donors (25-28 years old) unrelated to those who provided deciduous teeth. Procedures for handling human samples were approved by Kyushu University Institutional Review Board for Human Genome/Gene Research (Protocol Number: 393-00).

All animal experiments were approved by the Institutional Animal Care and Use Committee of Kyushu University (Protocol Number: A21-044-1; A25-086-0) and Nagoya University (Permissions \#28316, \#29121, \#30312, \#31199 and \#20430). Stem cell transplantation caused no substantial biological alterations or welfare issues in the animals (Fig. 4, Extended Data Fig. 8). All experiments were performed in accordance with relevant guidelines and regulations.

\section{Animals}

Male JF1 mice (National Institute of Genetics, Mishima, Japan) and male and female wild-type C57BL/6J (B6) mice (Kyudo, Tosu, Japan; Japan SLC, Hamamatsu, Japan) were housed under controlled 
environmental conditions (12-h light/12-h dark cycle) and given free access to sterile drinking water and a standard MF chow diet (Oriental Yeast, Tokyo, Japan).

\section{Stem cell isolation and culture}

Isolation and culture of stem cells from dental pulp tissue were performed using enzyme digestion and colony-forming unit fibroblast (CFU-F) methods ${ }^{14,15,42,43}$. Remnant dental pulp tissues from extracted human deciduous teeth were digested with $0.3 \%$ collagenase type I (Worthington Biochemicals, Lakewood, NJ, USA) and $0.4 \%$ dispase II (Sanko Junyaku Co., Ltd., Tokyo, Japan) for $60 \mathrm{~min}$ at $37^{\circ} \mathrm{C}$. The resultant cells were passed through a 70- $\mu \mathrm{m}$ cell strainer and seeded on T-75 culture flasks. Three hours after cell seeding, the culture flasks were washed with sterilized $\mathrm{Ca}^{2+}$-free and $\mathrm{Mg}^{2+}$-free phosphatebuffered saline (PBS). The remaining adherent cells were incubated in Minimum Essential Medium EagleAlpha Modification (aMEM; Thermo Fisher Scientific, Waltham, MA, USA) containing $15 \%$ foetal bovine serum (FBS; Equitech-Bio, Kerrville, TX, USA), $100 \mu \mathrm{M} \mathrm{L-ascorbic} \mathrm{acid} \mathrm{2-phosphate} \mathrm{(Wako} \mathrm{Pure} \mathrm{Chemicals,}$ Osaka, Japan), 2 mM L-glutamine (Nacalai Tesque, Kyoto, Japan) and premixed $100 \mathrm{U} / \mathrm{mL}$ penicillin/100 $\mu \mathrm{g} / \mathrm{mL}$ streptomycin (Nacalai Tesque) for 16 days. The resulting colonies were passaged to expand the number of dDPSCs. Cells at P3 were used for the experiments.

BMMSCs isolated from human whole bone marrow aspirates obtained from healthy adult volunteers $(n=$ 3; AllCells, Alameda, CA, USA) and human skin fibroblasts (FBs; PromoCell, Heidelberg, Germany) were prepared as described previously ${ }^{15}$.

\section{Stem cell characterization}

dDPSCs were characterized according to standard protocols ${ }^{44}$. The adherent colony-forming capacity was evaluated using a CFU-F assay. Isolated cells $\left(10^{\prime} 10^{3}\right)$ were seeded on T-75 culture flasks and cultured in growth medium for 16 days. The flasks were treated with $4 \%$ paraformaldehyde and $0.1 \%$ toluidine blue in PBS ( $\mathrm{pH} 7.4$ ) for 18 hours. The number of fibroblast colonies containing $>50$ cells was counted under a microscope.

The multipotency of dDPSCs (P3) was verified according to previous studies ${ }^{12,15}$. Briefly, specific culture conditions were used to induce dDPSCs to differentiate into osteoblasts, chondrocytes or adipocytes that were stained with alizarin red-S, toluidine blue or oil red-0, respectively. Expressions of genes specific to osteoblasts (runt-related transcription factor-2 [RUNX2] and osteocalcin [BGLAP]), chondrocytes (SRYbox-9 [SOX9] and aggrecan [ACAN]) and adipocytes (peroxisome proliferator-activated receptor gamma-2 [PPARG] and lipoprotein lipase [LPL]) were analysed by quantitative reverse transcription polymerase chain reaction (RT-qPCR). Expressions of genes specific for neural crest cells, including nerve growth factor receptor (NGFR), paired box-3 (PAX3), zinc finger protein SNAI-2 (SLUG), zinc finger protein SNAI-1 (SNAIL), SOX9 and endothelin receptor type B (EDNRB), in dDPSCs (P3) were examined by RT-qPCR. Expressions of CXCR4 and KIT in dDPSCs (P3) were assessed using immunofluorescence experiments (see Supplementary Table 1). We have previously confirmed that dDPSCs cultured under the same 
conditions express stem cell markers (e.g., CD146) and low levels of class II HLA and T cell costimulatory molecules ${ }^{15}$.

For mixed lymphocyte assays, human PBMNCs $\left(1 \times 10^{6}\right)$ were co-cultured with dDPSCs $\left(100 \times 10^{3}\right)$ and gamma-irradiated human PBMNCs $\left(100 \times 10^{3} ; 30\right.$ Gy irradiation with an MBR-1520R-3 irradiator; Hitachi, Tokyo, Japan) or stimulated with phytohemagglutinin ( $5 \mu \mathrm{g} / \mathrm{mL}$; Merck, Darmstadt, Germany) in RPMI1640 medium (Sigma-Aldrich) containing 10\% heat-inactivated FBS (Equitech-Bio), 2 mM L-glutamine, 1 $\mathrm{mM}$ sodium pyruvate and premixed $100 \mathrm{U} / \mathrm{mL}$ penicillin $/ 100 \mu \mathrm{g} / \mathrm{mL}$ streptomycin (Nacalai Tesque). Five days after co-culture or stimulation, cell viability was assayed using Cell Counting Kit-8 (Dojindo, Kumamoto, Japan) and a Multiscan GO plate reader (Thermo Fisher Scientific).

\section{Stem cell transplantation}

dDPSCs, BMMSCs or FBs ( $10^{4}$ cells/g bodyweight in $100 \mu \mathrm{L}$ PBS) were intravenously injected into JF1 mice at P11W. Age-matched B6 and JF1 mice intravenously injected with PBS $(100 \mu \mathrm{L})$ were used as controls. Some JF1 mice were injected intraperitoneally with anti-SDF1a antibody (100 $\mu \mathrm{g}$ in $100 \mu \mathrm{L}$ PBS; R\&D Systems, Minneapolis, MN, USA) or PBS $(100 \mu \mathrm{L}) 24$ hours before transplantation.

Mice were divided into five experimental groups: B6 $(n=10)$, (PBS-infused) JF1 $(n=10)$, dDPSCtransplanted JF1 $(n=10)$, BMMSC-transplanted JF1 $(n=10)$ and FB-transplanted JF1 $(n=10)$. Mouse bodyweight, water intake, food intake and stool number were measured weekly from P11W to P35W. Some mice were selected randomly for harvesting of organ tissues and peripheral blood at P23W (12 weeks after transplantation), and the intestine was dissected into lengths of $\sim 15 \mathrm{~mm}$.

\section{Monitoring of transplanted cells}

To reduce the background autofluorescence of standard chow, mice were fed with alfalfa-free Ivid\#2 feed (Oriental Yeast) for 10 days before in vivo and ex vivo imaging. dDPSCs were incubated with DiR (Perkin Elmer, Waltham, MA, USA) for 30 min at $37^{\circ} \mathrm{C}$ and washed twice with PBS. Fluorescent dDPSCs $\left(10^{5}\right.$ cells/g bodyweight in $100 \mu \mathrm{L}$ PBS) were intravenously transplanted into recipient mice (P23W). PBSinfused mice were used as controls. Whole-body images and ex vivo images of organs were acquired 1, 7 and 20 days after transplantation using an IVIS Lumina III system (Perkin Elmer). In the experiment involving the injection of anti-SDF1a antibody into a JF1 mouse, ex vivo imaging was performed 18 days after dDPSC transplantation due to early death of the mouse. Colour images showing DiR fluorescence above a threshold level were superimposed on greyscale images of the mouse body or resected organ.

\section{Peristalsis}

Mice were fed with standard chow, which exhibits autofluorescence. The entire gastrointestinal tract was resected (P23W) after a 24-hour fast. The autofluorescence of the intestinal contents was measured from ex vivo images acquired with an IVIS Lumina III system (Perkin Elmer). A reduction in autofluorescence level was interpreted as an increase in peristaltic movements. 


\section{Nutritional status}

Improvements in nutritional status (hepatic glucose uptake and body weight) after dDPSC transplantation were used as surrogate markers of mucosal repair in the small intestine. Tibial bones were harvested from each experimental group at P23W, and the distal tibial epiphyses were imaged using a Skyscan 1076 micro-CT system (Skyscan, Kontich, Belgium). Bone mineral density (BMD) and bone parameters were estimated using CT-Analyzer software (Skyscan). BMD values were calibrated using hydroxyapatite phantoms with BMD values of 0.25 and $0.75 \mathrm{~g} / \mathrm{cm}^{3}$ (Skyscan).

In vivo hepatic glucose uptake was measured in mice (P23W) given free access to water containing 1\% 2deoxy-D-glucose (2DG; Merck) ${ }^{45}$. Liver tissue samples were collected after 7 days. Hepatic 2DG content (an index of glucose storage) was assayed using a commercial kit (Cosmo Bio, Otaru, Japan) and plate reader (Multiscan GO; Thermo Fisher Scientific).

\section{Histology and immunohistochemistry}

Samples were fixed with $4 \%$ paraformaldehyde in PBS $(\mathrm{pH} 7.2)$ at $4^{\circ} \mathrm{C}$ overnight, dehydrated, cleaned and embedded in paraffin. For histology, sections were stained with haematoxylin-eosin. For immunohistochemistry, sections were incubated first with $3 \% \mathrm{H}_{2} \mathrm{O}_{2}$ in ethanol for 30 min to inhibit endogenous peroxidase and then with 10\% bovine serum albumin in PBS for 60 min. Sections were treated with $0.01 \mathrm{M}$ citrate buffer ( $\mathrm{pH} 6.0$ or 9.0) in a microwave processor (MI-77, Azumaya, Tokyo, Japan) for antigen retrieval. Sections were incubated with primary antibodies (Supplementary Table 2) at $4^{\circ} \mathrm{C}$ overnight. Visualisation was achieved using an EnVision+ System (Agilent, Santa Clara, CA, USA). Sections were lightly counterstained with haematoxylin. For double-immunofluorescence staining, sections were incubated with primary antibodies (Supplementary Table 2) and then treated with the Opal 3-plex kit (Perkin Elmer). Sections were mounted onto slides using Vectashield Mounting Medium with DAPI (Vector Laboratories, Burlingame, CA, USA). Negative controls utilized isotype-matched antibodies instead of primary antibodies. Images were obtained using a BZ-X700 fluorescence microscope (Keyence, Tokyo, Japan). The number and area of ganglion cells in myenteric ganglia were estimated using Hybrid Cell Count software (Keyence) ${ }^{46-48}$.

\section{Quantitative assays}

Blood samples were incubated overnight at $4^{\circ} \mathrm{C}$ and centrifuged at $1,500 \mathrm{rpm}$ for $10 \mathrm{~min}$ at $4^{\circ} \mathrm{C}$ to obtain serum. Tissue samples (including liver, lung and kidney) were homogenized in T-PER Tissue Protein Extraction Reagent (Thermo Fisher Scientific) containing a proteinase inhibitor cocktail (Nacalai Tesque) using a Vibra-Cell ultrasonic processor (Sonic \& Materials, Newtown, CT, USA). Total protein was measured using a protein assay (Bio-Rad Laboratories, Hercules, CA, USA) and normalized to the tissue sample weight.

Commercial kits were used to measure serum levels of creatinine (Creatinine Colorimetric Assay kit, Cayman Chemical Company, Ann Arbor, MI, USA), alanine aminotransferase and aspartate 
aminotransferase (Transaminase Cll-Test kit, Wako Pure Chemicals). Commercial enzyme-linked immunosorbent assay (ELISA) kits were used to determine albumin, GDNF, IL-2, MIP-2, NGF, SCF and SDF1a protein levels in mouse tissues or serum (see Supplementary Table 3). The colourimetric assay and ELISA results were analysed using a Multiscan GO plate reader (Thermo Fisher Scientific).

Serum levels of IL-1a, IL-1 $\beta$, IL-2, IL-5, IL-6, IL-12, IL-18, granulocyte-colony stimulating factor, tumour necrosis factor- $\alpha$, interferon- $\gamma$ and macrophage inflammatory protein-1 $\beta$ (MIP1 $\beta$ ) were quantified using a Bio-Plex MAGPIX system and a Bio-Plex Pro multiplex cytokine assay system (Bio-Rad Laboratories).

\section{RT-qPCR}

Samples were treated with TRIzol (Thermo Fisher Scientific). RNA extracts were digested with DNase I (Promega, Fitchburg, WI, USA) and purified using the RNeasy Mini Kit (Qiagen, Venlo, Netherlands). Total RNA samples were treated with a Revertra Ace qPCR kit (Toyobo, Osaka, Japan) to obtain cDNA. cDNA samples were mixed with EagleTaq Universal Master Mix (Roche, Basel, Switzerland) and target-specific TaqMan probes (Thermo Fisher Scientific; Supplementary Tables 4 and 5). RT-qPCR was performed using a Light Cycler 96 system (Roche) under the following conditions: $50^{\circ} \mathrm{C}$ for $120 \mathrm{sec} ; 95^{\circ} \mathrm{C}$ for $600 \mathrm{sec} ; 45$ cycles of $95^{\circ} \mathrm{C}$ for $15 \mathrm{sec}$ and $60^{\circ} \mathrm{C}$ for $60 \mathrm{sec}$. Glyceraldehyde 3-phosphate dehydrogenase mRNA (mouse samples) and $18 \mathrm{~S}$ ribosomal RNA (human samples) were used for data normalization.

\section{Mouse intestinal microbiota}

Stools were collected daily during P24W-P26W. Faecal bacterial DNA content was analysed by the Primary Cell Division of Cosmo Bio (Sapporo, Japan; protocol \#151228A1 and \#160125A1). Briefly, microbial DNA was extracted from the faecal sample using the Isospin Fecal DNA kit (Nippon Gene, Tokyo, Japan). The V3V4 region of the 16S rRNA gene was amplified by PCR (forward: CCTACGGGNGGCWGCAG; reverse: GACTACHVGGGTATCTAATCC) and sequenced using a MiSeq Deep sequencer and MiSeq Reagent Kit v3 (Illumina, San Diego, CA, USA). The sequence data were preprocessed and analysed using Flora Genesis software (Repertoire Genesis, Ibaraki, Japan). The R1 and R2 read pairs were joined, and chimera sequences were removed. Open-reference operational taxonomic unit (OTU) picking was performed using the $97 \%$ ID prefiltered Greengenes database and Uclust.

Representative sequences of each OTU were chosen, and taxonomy assignment was performed using the Ribosomal Database Project (RDP) classifier and a threshold score $\geq 0.5$. OTUs were grouped if their annotation was the same regardless of RDP score. Bacterial floral communities were characterised using the Gini-Simpson index (1/D) $)^{49,50}$.

\section{Colonic mechanical activity}

Mechanical responses to pharmacological agents and EFS were measured in 15-mm-long muscle strips isolated from the proximal colon. Macroscopically, the mouse proximal colon has two bulging regions; here we refer to the oral one as the first part and the anal one as the second part. The second part of the proximal colon was used in all experiments except those shown in Extended Data Figure $5 \mathrm{~g}$. The 
modified Krebs solution consisted of $121.6 \mathrm{mM} \mathrm{NaCl}, 4.7 \mathrm{mM} \mathrm{KCl}, 2.5 \mathrm{mM} \mathrm{CaCl}_{2}, 1.2 \mathrm{mM} \mathrm{MgCl}, 1.2 \mathrm{mM}$ $\mathrm{Na}_{2} \mathrm{KH}_{2} \mathrm{PO}_{4}, 15.4 \mathrm{mM} \mathrm{NaHCO}_{3}$ and $11.5 \mathrm{mM}$ D-glucose gassed with $5 \% \mathrm{CO}_{2}$ and $95 \% \mathrm{O}_{2}(\mathrm{pH} 7.4$ at $37^{\circ} \mathrm{C}$ ). The strips were mounted vertically in an organ bath maintained at $37^{\circ} \mathrm{C}$. Changes in isometric tension generated by the circular muscle were recorded using a force transducer (TB-612T; Nihon Kohden, Tokyo, Japan) and amplifier (AS1202; NEC, Tokyo, Japan) and analysed using LabChart and Scope software (ADInstruments, Bella Vista, NSW, Australia). Enteric nerve stimulation was achieved using EFS ( $50 \mathrm{~V}, 0.5$-msec step pulses repeated at $5 \mathrm{~Hz}$ for $5 \mathrm{sec}$ ) applied by an electrical stimulator (SEN-3301; Nihon Kohden). The following pharmacological agents were used: $1 \mu \mathrm{M}$ Ach (Wako Pure Chemicals), 10 nM ET-1 (Wako Pure Chemicals), $10 \mu \mathrm{M}$ NO (Wako Pure Chemicals), $60 \mathrm{mM} \mathrm{KCl,} 1 \mu \mathrm{M}$ atropine sulphate (Wako Pure Chemicals; to block the response to Ach) and $1 \mu \mathrm{M}$ tetrodotoxin (Wako Pure Chemicals; to block the response to EFS). Tension development in response to EFS and pharmacological agents was normalized to the tissue weight and response to $60 \mathrm{mM} \mathrm{KCl}$.

\section{Colonic electrical activity}

The spatiotemporal characteristics of proximal colon electrical activity were assessed using an $8 \times 8$ MEA recording system (Alpha MED Scientific, Ibaraki, Japan) ${ }^{51,52}$. Colonic smooth muscle samples were prepared by gently removing the mucosa. Each sample was mounted on an $8 \times 8$ MEA (interpolar distance, $150 \mu \mathrm{m}$ ) with the longitudinal muscle layer facing downward using a piece of dialysis membrane fixed with a slice anchor (SDH series, Harvard Apparatus Japan, Tokyo, Japan; Extended Data Figure 6). The 2$\mathrm{mL}$ recording chamber was perfused $\left(1-2 \mathrm{~mL} / \mathrm{min}\right.$ at $\left.34^{\circ} \mathrm{C}\right)$ with modified Krebs solution containing HEPES/Tris pH-buffer instead of bicarbonate to prevent the development of microbubbles on the MEA. The sample was perfused with solution for $\sim 30$ min before recording was started, and perfusion was discontinued during recording to minimize electrical noise. A set of $8 \times 8$ field potentials were simultaneously recorded using a computer-controlled, multi-channel AC amplifier (low-pass filtering at 10 $\mathrm{kHz}$ ) and 14-bit A/D converters (sampling rate of $20 \mathrm{kHz}$ ). The dynamic range of $A / D$ conversion was usually $\pm 1 \mathrm{mV}$. High-pass $(0.1 \mathrm{~Hz})$ filtering was applied to stabilize the baseline drift of the microelectrode potential.

A low-impedance MEA was employed to measure a wide range of electrical activity, including pacemaker potentials occurring at $\sim 4$-sec intervals and myoelectric complex-like potentials with rapid and slow components ${ }^{51}$. Each recording electrode was a square $(\sim 50 \mu \mathrm{m} \times 50 \mu \mathrm{m})$ made from platinum black nanoparticles, which increased the surface area by $\sim 200$-fold to $0.5 \mathrm{~mm}^{2}$. The capacitance (CME) and resistance (RME) of each microelectrode was $0.052 \mu \mathrm{F}$ and $15 \mathrm{k} \Omega$, respectively ${ }^{51,53}$, hence the impedance of the recording electrode at $0.1 \mathrm{~Hz}$ was estimated to be small enough $[\sim 31 \mathrm{M} \Omega=\sqrt{ }\{1 /(2 \pi \times 0.1 \mathrm{~Hz} \times$ $\left.0.052 \mu \mathrm{F})^{2}+(15 \mathrm{k} \Omega)^{2}\right\}$ ] to follow oscillating potentials when compared to the input impedance of the multi-channel amplifier (100 M $\Omega$ at $0.1 \mathrm{~Hz}$ ). The efficacy of electrical signal transmission (Tr) was estimated to be $\sim 95 \%$ at $0.1 \mathrm{~Hz}\left[100 \mathrm{M} \Omega / \sqrt{ }\left\{(100 \mathrm{M} \Omega)^{2}+(31 \mathrm{M} \Omega)^{2}\right\}\right]$. Moreover, the low resistance (15 $\mathrm{k} \Omega$ ) of the electrodes was advantageous for reducing thermal noise (NT), which was calculated to be $\sim 1.6 \mu \mathrm{V}$ at the low-pass frequency $(10 \mathrm{kHz})$ of the $\mathrm{AC}$ amplifier. 
To generate pseudocolour images, the field potential data were thinned 100-1000-fold in the time domain and bandpass filtered at $0.25-30 \mathrm{~Hz}$ (Kaiseki Excel add-in software, Kyowa, Tokyo, Japan). At certain recording times, the $8 \times 8$ field potentials were interpolated by a spline function with 50 points between each potential using MATLAB (MathWorks, Natick, MA, USA) ${ }^{53}$. To reconstitute smooth potential images, the amplitude of the field potential in each ME was compensated by the linear spectrum at 0.1$15 \mathrm{~Hz}^{51}$. The upper and lower images corresponded to the oral and anal ends of the MEA recording region, respectively. Field potential videos were produced at a frame rate of $200 \mathrm{~Hz}$.

\section{Statistical analysis}

Data are expressed as the mean \pm standard error of the mean (SEM) of at least three determinations. Inter-group comparisons were made using two-tailed Student's t-tests for distinct samples (two groups), Mann-Whitney U-test or one-way repeated-measures analysis of variance (ANOVA) followed by the Tukey post-hoc test (three or more groups). Kaplan-Meier analyses and log-rank tests were used for survival analyses. $P<0.05$ was considered significant. Analyses were performed using JMP 11 (SAS Institute, Cary, NC, USA).

\section{Declarations}

\section{Approval}

All authors (K.Y., T.Y., S.K., S.S., Y.Y., T.M., J.Y., Y.O., N.I., C.T., S.N. and T.T.) approved the manuscript.

\section{Competing interests}

The authors declare no competing interests.

\section{Contributors}

Tomoaki Taguchi (1) conceived the idea of dDPSC-based therapy for entero-'neuropathy', and Shinsuke Nakayama (2) conceived the idea of co-impairment of the pacemaker system in entero-'neuropathy'. Takayoshi Yamaza (3), Shunichi Kajioka (4) and S.N. designed the study. T.Y. and Soichiro Sonoda (2) characterized and transplanted the stem cells. Koichiro Yoshimaru (1), Yusuke Yanagi (1), Toshiharu Matsuura (1) Yoshinao Oda (4) and S.K. performed the histological studies, enzyme assays, geneexpression studies, contractile force studies and in vivo and ex vivo imaging and corresponding data curation. Naoko Iwata (2), Chiho Takai (2), Junko Yoshizumi (6) and S.N. performed the electrophysiology experiments, data curation and visualization of the data. Haruyoshi Yamaza (6) collected teeth samples. Eiji Mitate (7) supported the analysis of electrophysiology data. Ratih Yuniartha (1), Tomoko Yamazaki (1), Kozue Urata (1), Rie Funatsu (1), Jumi Matsumoto (5) and Erika Tomoda (3) supported histological studies, enzyme assays, gene-expression studies, in vivo and ex vivo imaging and animal management.

S.K. performed the statistical analysis of all the source data, and S.K. and S.N. constructed the figures. S.N. wrote the manuscript using the data from S.K. with the input from contributors. 
Address of contributors:

(1) Department of Pediatric Surgery, Kyushu University Graduate School of Medical Sciences, Fukuoka, Japan.

(2) Department of Cell Physiology, Nagoya University Graduate School of Medicine, Nagoya, Japan.

(3) Department of Molecular Cell Biology and Oral Anatomy, Kyushu University Graduate School of Dental Science, Fukuoka, Japan.

(4) Department of Clinical Pharmacology, Kyushu University Graduate School of Medical Sciences, Fukuoka, Japan.

(5) Department of Anatomic Pathology, Kyushu University Graduate School of Medical Sciences, Fukuoka, Japan.

(6) Department of Pediatric Dentistry, Kyushu University Graduate School of Dental Science, Fukuoka, Japan.

(7) Department of Regenerative Oral Surgery, Unit of Translational Medicine, Nagasaki University, Japan

\section{Acknowledgements}

The authors thank OXMEDCOMMS (www.oxmedcomms.com) for assistance with the preparation of the manuscript, and contributors for experimentation and data curation.

The authorship of the contributors toward the submission of journals' format is under assessment.

\section{Funding}

This work was supported by Grant-in-Aid for Scientific Research from the Japan Society for the Promotion Science (JSPS): 25253094 (T.T., K.Y, T.Y., S.K.), 15K10922 (K.Y.) and $19 H 03558$ (N.I., C.T., S.N.); Grant-in-Aid for Challenging Exploratory Research Project from JSPS 25670744 (T.T.); Research grant from the Ministry of Health, Labor and Welfare H26-040 (T.T.); Research grant from the Japan Agency for Medical Research and Development (AMED) 16ek0210037h0003 (S.N.); Research grant The Japan Foundation for Pediatric Research 15-002 (K.Y.); Research grant from Suzuken Memorial Foundation (S.N., N.I., C.T.).

\section{Data availability}

The source data for the text and figures of this study are available from the authors upon reasonable request.

\section{References}


1. Burns, A. J., Goldstein, A. M., Newgreen, D. F., Stamp, L., Schäfer, K. H., Metzger, M., Hotta, R., Young, H. M., Andrews, P. W., Thapar, N., Belkind-Gerson, J., Bondurand, N., Bornstein, J. C., Chan, W. Y., Cheah, K., Gershon, M. D., Heuckeroth, R. O., Hofstra, R. M., Just, L., Kapur, R. P., King, S. K., McCann, C. J., Nagy, N., Ngan, E., Obermayr, F., Pachnis, V., Pasricha, P. J., Sham, M. H., Tam, P. \& Vanden Berghe, P. White paper on guidelines concerning enteric nervous system stem cell therapy for enteric neuropathies. Dev. Biol. 417, 229-251 (2016). doi:10.1016/j.ydbio.2016.04.001 2. Watanabe, Y., Kanamori, Y., Uchida, K. \& Taguchi, T. Isolated hypoganglionosis: results of a nationwide survey in Japan. Pediatr. Surg. Int. 29, 1127-1130 (2013). doi:10.1007/s00383-013-3378-5 3. Taguchi, T., Ieiri, S., Miyoshi, K., Kohashi, K., Oda, Y., Kubota, A., Watanabe, Y., Matsufuji, H., Fukuzawa, M. \& Tomomasa, T. The incidence and outcome of allied disorders of Hirschsprung's disease in Japan: Results from a nationwide survey. Asian J. Surg. 40, 29-34 (2017). doi:10.1016/j.asjsur.2015.04.004 4. Heuckeroth, R. O. Hirschsprung disease - integrating basic science and clinical medicine to improve outcomes. Nat. Rev. Gastroenterol. Hepatol. 15, 152-167 (2018). doi:10.1038/nrgastro.2017.149 5. Di Lorenzo, C., Solzi, G. F., Flores, A. F., Schwankovsky, L. \& Hyman, P. E. Colonic motility after surgery for Hirschsprung's disease. Am J Gastroenterol. 95, 1759-1764 (2000). doi:10.1111/j.1572-0241.2000.02183.x 6. Hirschsprung, H. Stuhlträgheit Neugeborener in Folge von Dilatation und Hypertrophie des Colons. Jahrb. f. Kinderh. 27, 1-7 (1888). (See also:

https://en.wikipedia.org/wiki/Hirschsprung\%27s_disease). 7. Furness, J. B. The enteric nervous system and neurogastroenterology. Nat. Rev. Gastroenterol. Hepatol. 9, 286-294 (2012). doi:10.1038/nrgastro.2012.32 8. Huizinga, J. D., Thuneberg, L., Klüppel, M., Malysz, J., Mikkelsen, H. B. \& Bernstein, A. W/kit gene required for interstitial cells of Cajal and for intestinal pacemaker activity. Nature 373, 347-349 (1995). doi:10.1038/373347a0 9. Nakayama, S., Shimono, K., Liu, H. N., Jiko, H., Katayama, N., Tomita, T. \& Goto, K. Pacemaker phase shift in the absence of neural activity in guinea-pig stomach: a microelectrode array study. J. Physiol. 576, 727-738 (2006).

doi:10.1113/jphysiol.2006.118893 10. Huizinga, J. D. \& Lammers, W. J. Gut peristalsis is governed by a multitude of cooperating mechanisms. Am. J. Physiol. Gastrointest. Liver Physiol. 296, G1-G8 (2009). doi:10.1152/ajpgi.90380.2008 11. Attié, T., Pelet, A., Edery, P., Eng, C., Mulligan, L. M., Amiel, J., Boutrand, L., Beldjord, C., Nihoul-Fékété, C. \& Munnich, A. Diversity of RET proto-oncogene mutations in familial and sporadic Hirschsprung disease. Hum. Mol. Genet. 4, 1381-1386 (1995). doi:10.1093/hmg/4.8.1381 12. Miura, M., Gronthos, S., Zhao, M., Lu, B., Fisher, L. W., Robey, P. G. \& Shi, S. SHED: stem cells from human exfoliated deciduous teeth. Proc. Natl. Acad. Sci. U. S. A. 100, 5807-5812 (2003). doi:

10.1073/pnas.0937635100. 13. Chai, Y., Jiang, X., Ito, Y., Bringas, P., Jr, Han, J., Rowitch, D. H., Soriano, P., McMahon, A. P. \& Sucov, H. M. Fate of the mammalian cranial neural crest during tooth and mandibular morphogenesis. Development 127, 1671-1679 (2000). 14. Xuan, K., Li, B., Guo, H., Sun, W., Kou, X., He, X., Zhang, Y., Sun, J., Liu, A., Liao, L., Liu, S., Liu, W., Hu, C., Shi, S. \& Jin, Y. Deciduous autologous tooth stem cells regenerate dental pulp after implantation into injured teeth. Sci. Transl. Med. 10, eaaf3227 (2018). doi:10.1126/scitransImed.aaf3227 15. Iwanaka, T., Yamaza, T., Sonoda, S., Yoshimaru, K., Matsuura, T., Yamaza, H., Ohga, S., Oda, Y. \& Taguchi, T. A model study for the manufacture and validation of clinicalgrade deciduous dental pulp stem cells for chronic liver fibrosis treatment. Stem Cell Res. Ther. 11, 134 (2020). doi:10.1186/s13287-020-01630-w 16. Koide, T., Moriwaki, K., Uchida, K., Mita, A., Sagai, T., Yonekawa, H., Katoh, H., Miyashita, N., Tsuchiya, K., Nielsen, T. J. \& Shiroishi, T. A new inbred strain JF1 
established from Japanese fancy mouse carrying the classic piebald allele [published correction appears in Mamm. Genome 9, 344 (1998)]. Mamm. Genome 9, 15-19 (1998). doi:10.1007/s003359900672 17. Yamada, T., Ohtani, S., Sakurai, T., Tsuji, T., Kunieda, T. \& Yanagisawa, M. Reduced expression of the endothelin receptor type $B$ gene in piebald mice caused by insertion of a retroposon-like element in intron 1. J. Biol. Chem. 281, 10799-10807 (2006). doi:10.1074/jbc.M512618200 18. Dang, R., Sasaki, N., Torigoe, D. \& Agui, T. Anatomic modifications in the enteric nervous system of JF1 mice with the classic piebald mutation. J. Vet. Med. Sci. 74, 391-394 (2012). doi:10.1292/jvms.11-0447 19. Hosoda, K., Hammer, R. E., Richardson, J. A., Baynash, A. G., Cheung, J. C., Giaid, A. \& Yanagisawa, M. Targeted and natural (piebald-lethal) mutations of endothelin-B receptor gene produce megacolon associated with spotted coat color in mice. Cell 79, 1267-1276 (1994). doi:10.1016/0092-8674(94)90017-5 20. Pierre, J. F., Barlow-Anacker, A. J., Erickson, C. S., Heneghan, A. F., Leverson, G. E., Dowd, S. E., Epstein, M. L., Kudsk, K. A. \& Gosain, A. Intestinal dysbiosis and bacterial enteroinvasion in a murine model of Hirschsprung's disease. J. Pediatr. Surg. 49, 1242-1251 (2014). doi:10.1016/j.jpedsurg.2014.01.060 21. Kitaori, T., Ito, H., Schwarz, E. M., Tsutsumi, R., Yoshitomi, H., Oishi, S., Nakano, M., Fujii, N., Nagasawa, T. \& Nakamura, T. Stromal cell-derived factor 1/CXCR4 signaling is critical for the recruitment of mesenchymal stem cells to the fracture site during skeletal repair in a mouse model. Arthritis Rheum. 60, 813-823 (2009).

doi:10.1002/art.24330 22. Wedel, T., Spiegler, J., Soellner, S., Roblick, U. J., Schiedeck, T. H., Bruch, H. P. \& Krammer, H. J. Enteric nerves and interstitial cells of Cajal are altered in patients with slow-transit constipation and megacolon. Gastroenterology 123, 1459-1467 (2002). doi:10.1053/gast.2002.36600 23. Burns, A. J. Disorders of interstitial cells of Cajal. J. Pediatr. Gastroenterol. Nutr. 45 Suppl 2, S103S106 (2007). doi:10.1097/MPG.0b013e31812e65e0 24. Huizinga, J. D. \& White, E. J. Progenitor cells of interstitial cells of Cajal: on the road to tissue repair. Gastroenterology 134, 1252-1254 (2008). doi:10.1053/j.gastro.2008.02.074 25. Szurszewski, J. H. Electrical basis for gastrointestinal motility. In Physiology of Gastrointestinal Tract, 2nd edition (ed. Johnson, L.R.) 383-422 (Raven Press, New York, 1987). 26. Wood, J. D. Electrical activity of the intestine of mice with hereditary megacolon and absence of enteric ganglion cells. Am. J. Dig. Dis. 18, 477-488 (1973). doi:10.1007/BF01076598 27. Sarna, S. K. Myoelectric correlates of colonic motor complexes and contractile activity. Am. J. Physiol. 250, G213G220 (1986). doi:10.1152/ajpgi.1986.250.2.G213 28. Spencer, N. J. \& Bywater, R. A. Enteric nerve stimulation evokes a premature colonic migrating motor complex in mouse. Neurogastroenterol. Motil. 14, 657-665 (2002). doi:10.1046/j.1365-2982.2002.00367.x 29. Sakai, K., Yamamoto, A., Matsubara, K., Nakamura, S., Naruse, M., Yamagata, M., Sakamoto, K., Tauchi, R., Wakao, N., Imagama, S., Hibi, H., Kadomatsu, K., Ishiguro, N. \& Ueda, M. Human dental pulp-derived stem cells promote locomotor recovery after complete transection of the rat spinal cord by multiple neuro-regenerative mechanisms. J. Clin. Invest. 122, 80-90 (2012). doi:10.1172/JCl59251 30. Shimojima, C., Takeuchi, H., Jin, S., Parajuli, B., Hattori, H., Suzumura, A., Hibi, H., Ueda, M. \& Yamamoto, A. Conditioned medium from the stem cells of human exfoliated deciduous teeth ameliorates experimental autoimmune encephalomyelitis. J. Immunol. 196, 4164-4171 (2016). doi:10.4049/jimmunol.1501457 31. Mabuchi, Y., Morikawa, S., Harada, S., Niibe, K., Suzuki, S., Renault-Mihara, F., Houlihan, D. D., Akazawa, C., Okano, H. \& Matsuzaki, Y. LNGFR(+)THY1(+)VCAM-1 (hi+) cells reveal functionally distinct subpopulations in mesenchymal stem cells. Stem Cell Reports 1, 152-165 (2013). doi:10.1016/j.stemcr.2013.06.001 32. Hotta R, Stamp LA, Foong JP, et al. 
Transplanted progenitors generate functional enteric neurons in the postnatal colon. J Clin Invest. 2013;123(3):1182-1191. doi:10.1172/JCl65963 33. Workman MJ, Mahe MM, Trisno S, et al. Engineered human pluripotent-stem-cell-derived intestinal tissues with a functional enteric nervous system. Nat Med. 2017;23(1):49-59. doi:10.1038/nm.4233 34. Takaki M, Nakayama S, Misawa H, Nakagawa T, Kuniyasu H. In vitro formation of enteric neural network structure in a gut-like organ differentiated from mouse embryonic stem cells. Stem Cells. 2006;24(6):1414-1422. doi:10.1634/stemcells.2005-0394 35. Fattahi F, Steinbeck JA, Kriks S, et al. Deriving human ENS lineages for cell therapy and drug discovery in Hirschsprung disease. Nature. 2016;531(7592):105-109. doi:10.1038/nature16951 . 36. Zhao T, Zhang ZN, Rong Z, Xu Y. Immunogenicity of induced pluripotent stem cells. Nature. 2011;474(7350):212-215. doi:10.1038/nature10135 37. Araki R, Uda M, Hoki Y, et al. Negligible immunogenicity of terminally differentiated cells derived from induced pluripotent or embryonic stem cells. Nature.

2013;494(7435):100-104. doi:10.1038/nature11807 38. Shen WC, Lai YC, Li LH, et al. Methylation and PTEN activation in dental pulp mesenchymal stem cells promotes osteogenesis and reduces oncogenesis. Nat Commun. 2019;10(1):2226. doi:10.1038/s41467-019-10197-x 39. Lies, B., Beck, K., Keppler, J., Saur, D., Groneberg, D., Friebe, A. Nitrergic signalling via interstitial cells of Cajal regulates motor activity in murine colon. J. Physiol. 593, 4589-4601 (2015). doi:10.1113/JP270511 40. Ro S, Hwang SJ, Muto M, Jewett WK, Spencer NJ. Anatomic modifications in the enteric nervous system of piebald mice and physiological consequences to colonic motor activity. Am J Physiol Gastrointest Liver Physiol. 2006;290(4):G710-G718. doi:10.1152/ajpgi.00420.2005 41. Chatterjee S, Nandakumar P, Auer DR, Gabriel SB, Chakravarti A. Gene- and tissue-level interactions in normal gastrointestinal development and Hirschsprung disease. Proc Natl Acad Sci U S A. 2019;116(52):26697-26708. doi:10.1073/pnas.1908756116 Methods references 42. Ma, L., Makino, Y., Yamaza, H., Akiyama, K., Hoshino, Y., Song, G., Kukita, T., Nonaka, K., Shi, S. \& Yamaza, T. Cryopreserved dental pulp tissues of exfoliated deciduous teeth is a feasible stem cell resource for regenerative medicine. PLoS One 7, e51777 (2012). doi:10.1371/journal.pone.0051777 43. Yamaza, T., Alatas, F. S., Yuniartha, R., Yamaza, H., Fujiyoshi, J. K., Yanagi, Y., Yoshimaru, K., Hayashida, M., Matsuura, T., Aijima, R., Ihara, K., Ohga, S., Shi, S., Nonaka, K. \& Taguchi, T. In vivo hepatogenic capacity and therapeutic potential of stem cells from human exfoliated deciduous teeth in liver fibrosis in mice. Stem Cell Res. Ther. 6, 171 (2015). doi:10.1186/s13287-015-0154-6 44. Dominici, M., Le Blanc, K., Mueller, I., Slaper-Cortenbach, I., Marini, F., Krause, D., Deans, R., Keating, A., Prockop, D. J. \& Horwitz, E. Minimal criteria for defining multipotent mesenchymal stromal cells. The International Society for Cellular Therapy position statement.

Cytotherapy 8, 315-317 (2006). doi:10.1080/14653240600855905 45. Saito, K., Lee, S., Shiuchi, T., Toda, C., Kamijo, M., Inagaki-Ohara, K., Okamoto, S. \& Minokoshi, Y. An enzymatic photometric assay for 2deoxyglucose uptake in insulin-responsive tissues and 3T3-L1 adipocytes. Anal. Biochem. 412, 9-17 (2011). doi:10.1016/j.ab.2011.01.022 46. White, F. V. \& Langer, J. C. Circumferential distribution of ganglion cells in the transition zone of children with Hirschsprung disease. Pediatr. Dev. Pathol. 3, 216222 (2000). doi:10.1007/s100249910028 47. Swaminathan, M. \& Kapur, R. P. Counting myenteric ganglion cells in histologic sections: an empirical approach [published correction appears in Hum. Pathol. 43, 465 (2012)]. Hum. Pathol. 41, 1097-1108 (2010). doi:10.1016/j.humpath.2009.12.012 48. Yoshimaru, K., Taguchi, T., Obata, S., Takemoto, J., Takahashi, Y., Iwanaka, T., Yanagi, Y., Kuda, M., 
Miyoshi, K., Matsuura, T., Kinoshita, Y., Yoshioka, T., Nakazawa, A. \& Oda, Y. Immunostaining for Hu C/D and CD56 is useful for a definitive histopathological diagnosis of congenital and acquired isolated hypoganglionosis. Virchows Arch. 470, 679-685 (2017). doi:10.1007/s00428-017-2128-9 49. Zhou, J., Xia, B., Treves, D. S., Wu, L. Y., Marsh, T. L., O'Neill, R. V., Palumbo, A. V. \& Tiedje, J. M. Spatial and resource factors influencing high microbial diversity in soil. Appl. Environ. Microbiol. 68, 326-334 (2002).

doi:10.1128/aem.68.1.326-334.2002 50. van Nood, E., Vrieze, A., Nieuwdorp, M., Fuentes, S., Zoetendal, E. G., de Vos, W. M., Visser, C. E., Kuijper, E. J., Bartelsman, J. F., Tijssen, J. G., Speelman, P., Dijkgraaf, M. G. \& Keller, J. J. Duodenal infusion of donor feces for recurrent Clostridium difficile. N. Engl. J. Med. 368, 407415 (2013). doi:10.1056/NEJMoa1205037 51. Iwata, N., Fujimura, T., Takai, C., Odani, K., Kawano, S. \& Nakayama, S. Dialysis membrane-enforced microelectrode array measurement of diverse gut electrical activity. Biosens. Bioelectron. 94, 312-320 (2017). doi:10.1016/j.bios.2017.03.002 52. Morishita, H., Iwata, N., Takai, C., Mochizuki, N., Kaji, N., Hori, M., Kajioka, S. \& Nakayama, S. Micro-coordination of pacemaker potentials in the intestine of the mouse. Gastroenterology 152, 1831-1833.e4 (2017). doi:10.1053/j.gastro.2017.04.016 53. Taniguchi, M., Kajioka, S., Shozib, H. B., Sawamura, K., Nakayama, S. Spatial analysis of slowly oscillating electric activity in the gut of mice using low impedance arrayed microelectrodes. PLoS One 8, e75235 (2013). doi:10.1371/journal.pone.0075235

\section{Figures}


a

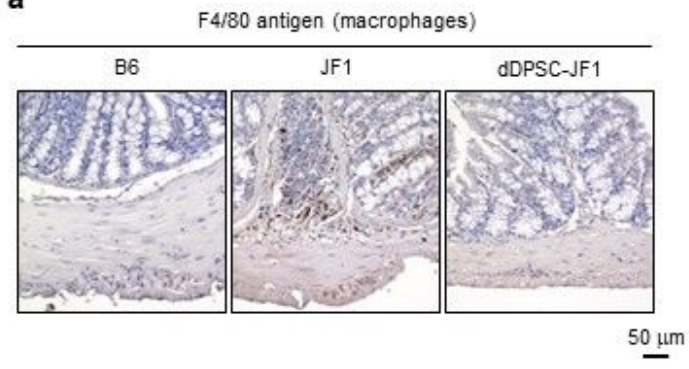

d
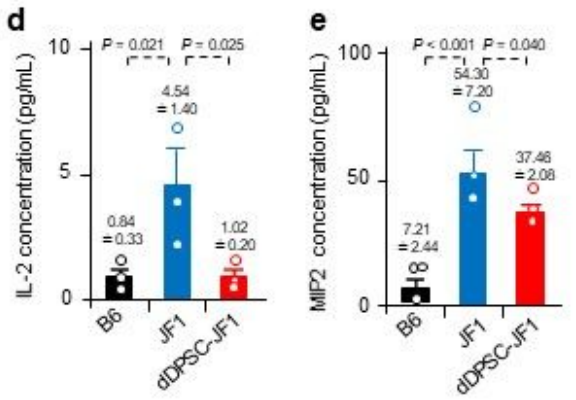

g

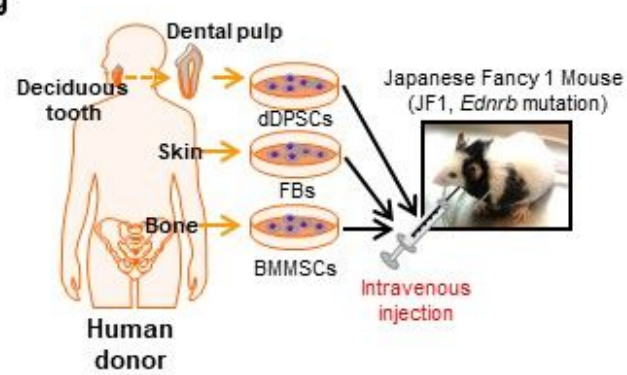

Injection Evaluation

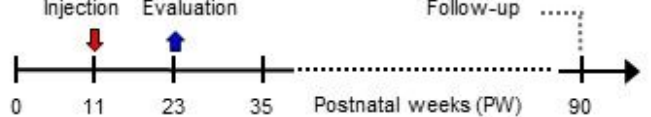

j

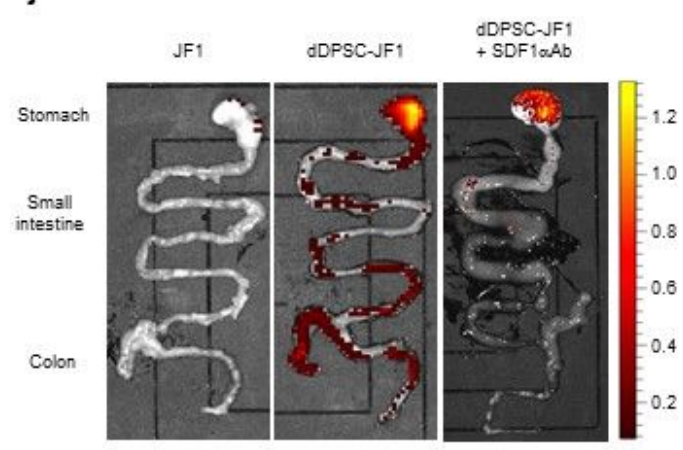

b
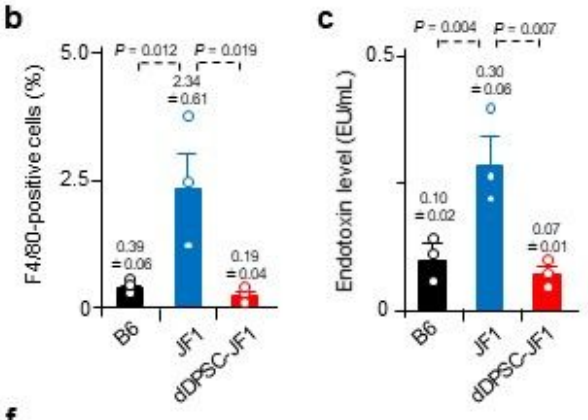

f

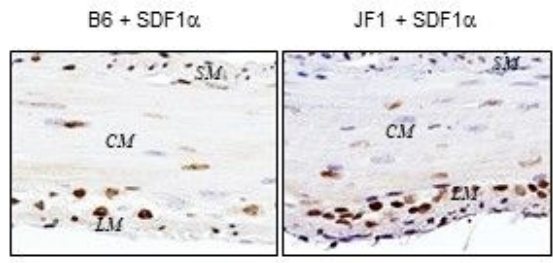

$50 \mu \mathrm{m}$

h
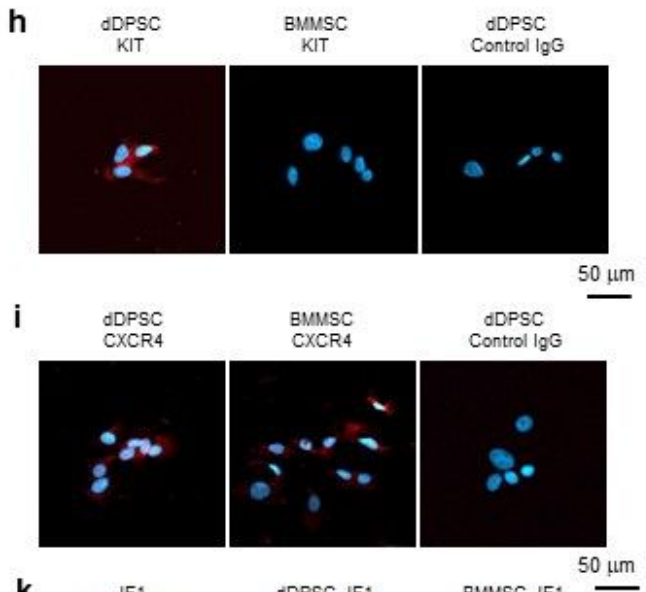

k

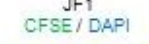

CFSE/ DAPI

CFSE / DAP
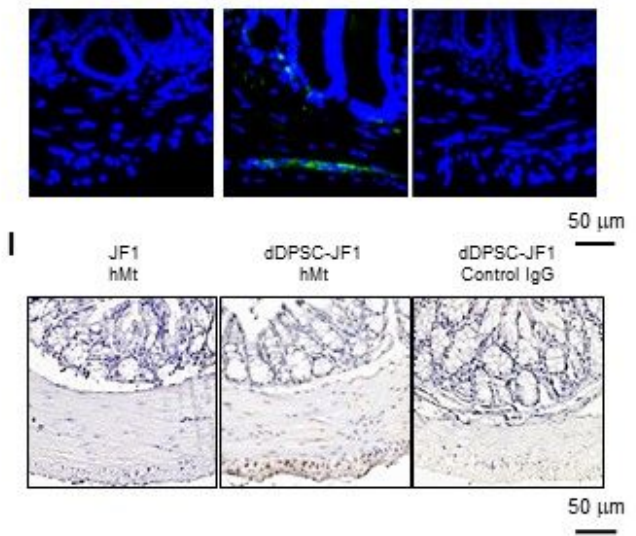

\section{Figure 1}

Intestinal migration of dDPSCs. a, Macrophages in the proximal colon. b, Macrophage percentage in the proximal colon (mean $\pm S E M, n=3$ ). $c-e$, Intestinal endotoxin, IL-2 and MIP2 levels (mean $\pm S E M, n=3$ ). $f$, Proximal colon SDF1a expression (×200). CM: circular muscle; LM: Iongitudinal muscle; SM: submucosal layer. g, Experimental schedule. h,i, Stem cell KIT and CXCR4 expression. j, Mouse intestine (ex vivo fluorescence imaging; $n=1$ ). k, CFSE-labelled cells 3 days post-transplantation (proximal colon, $\times 200$ ). 
Representative of $\geq 3$ experiments. I, hMt-containing cells 12 weeks post-transplantation (proximal colon, $\times 200)$. a, f, h, i, k, l: Representative of $\geq 2$ independent experiments.

a

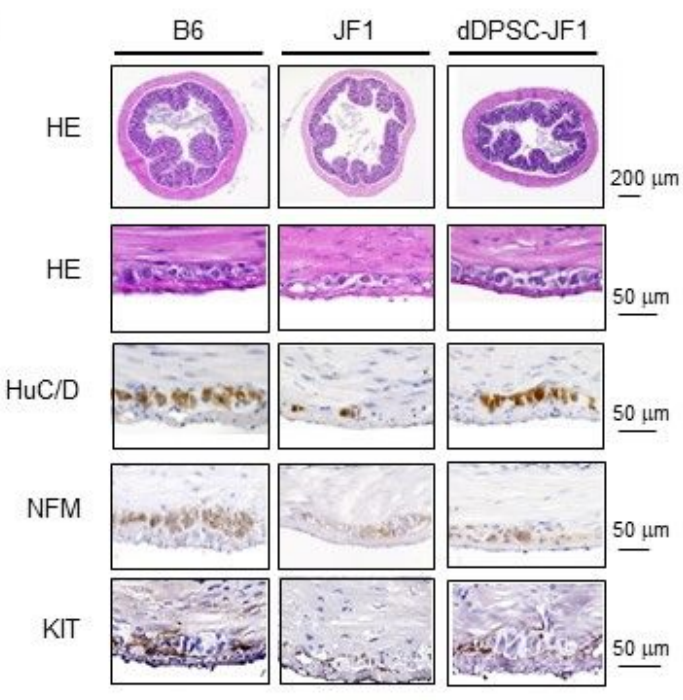

c

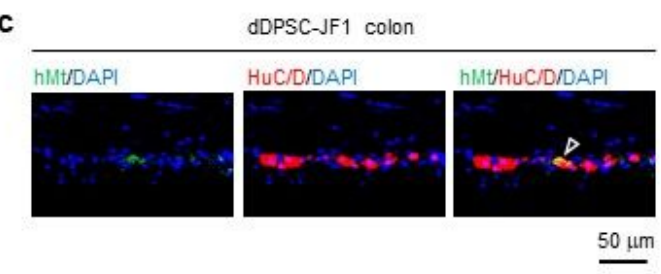

d

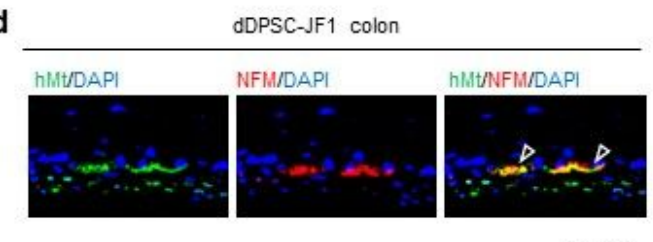

$50 \mu \mathrm{m}$

e

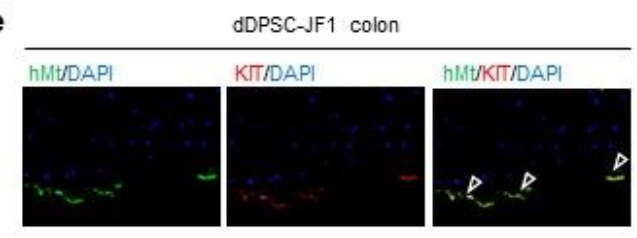

$50 \underline{\mu m}$
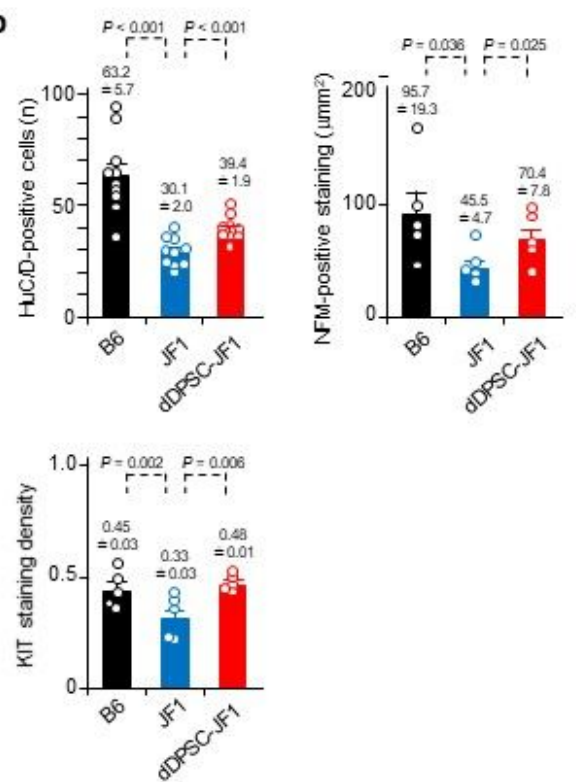

b

$\square^{\text {human }}$
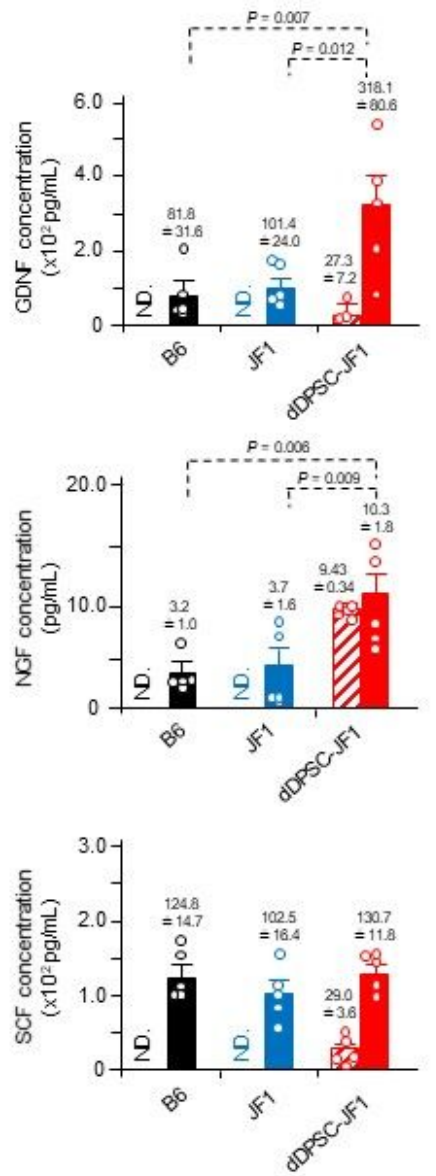

Figure 2

Differentiation of transplanted dDPSCs. a, Proximal colon sections stained with haematoxylin-eosin (upper: $\times 40$; lower: $\times 400$ ) or immunostained for HuC/D (neuron-specific marker; $\times 400$ ), NFM (peripheral ganglion marker; $\times 200$ ) or KIT (pacemaker interstitial cell marker; $\times 200$ ). b, Number of HuC/D-positive 
cells per tissue cross-section (mean $\pm S E M, n=10$ ), NFM-positive area in the myenteric plexus (mean $\pm S E M$, $\mathrm{n}=5$ ), and KIT-positive staining density in the proximal colon (mean $\pm S E M, n=5$ ). $c-e$, Immunostaining for hMt, HuC/D, NFM and KIT (nuclei counterstained with DAPI). Arrowheads: dDPSC-derived cells. f, Human and mouse NGF, GDNF and SCF concentrations (mean \pm SEM, $n=5$ ). ND, not detected. a, c-e:

Representative of $\geq 2$ independent experiments.
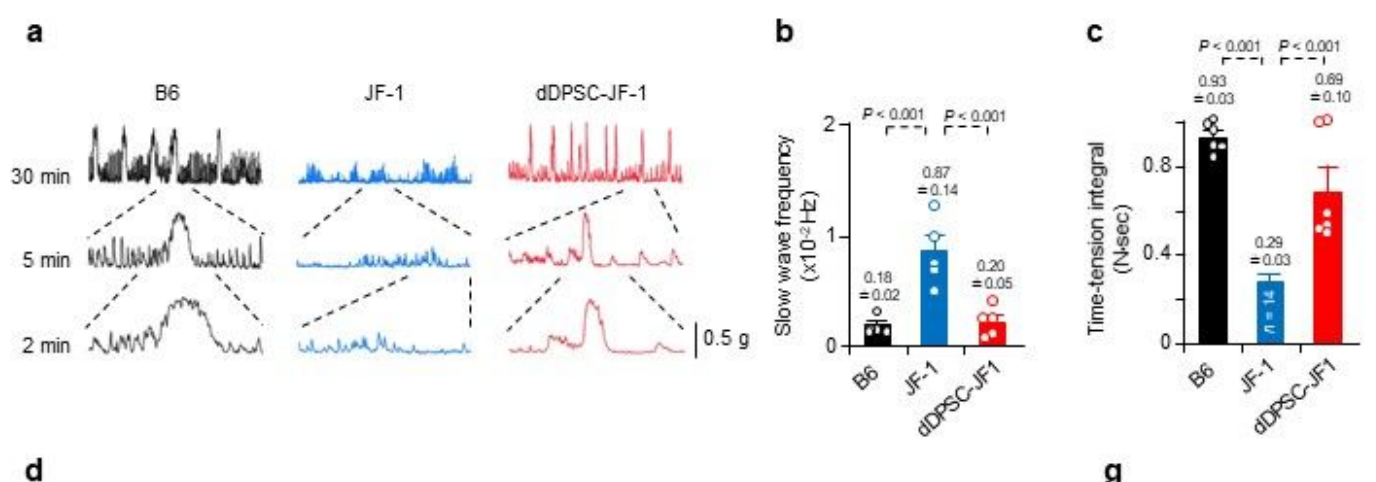

$\mathrm{B} 6$

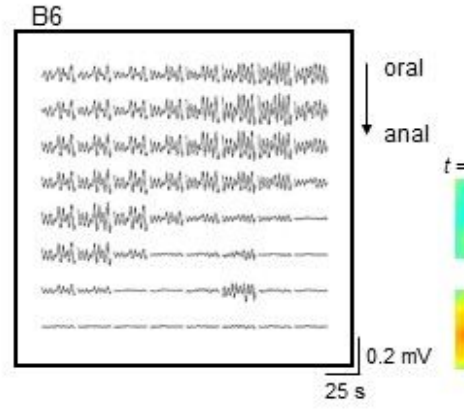

ME6 (blue), ME22 (red), ME38 (green)

g

e
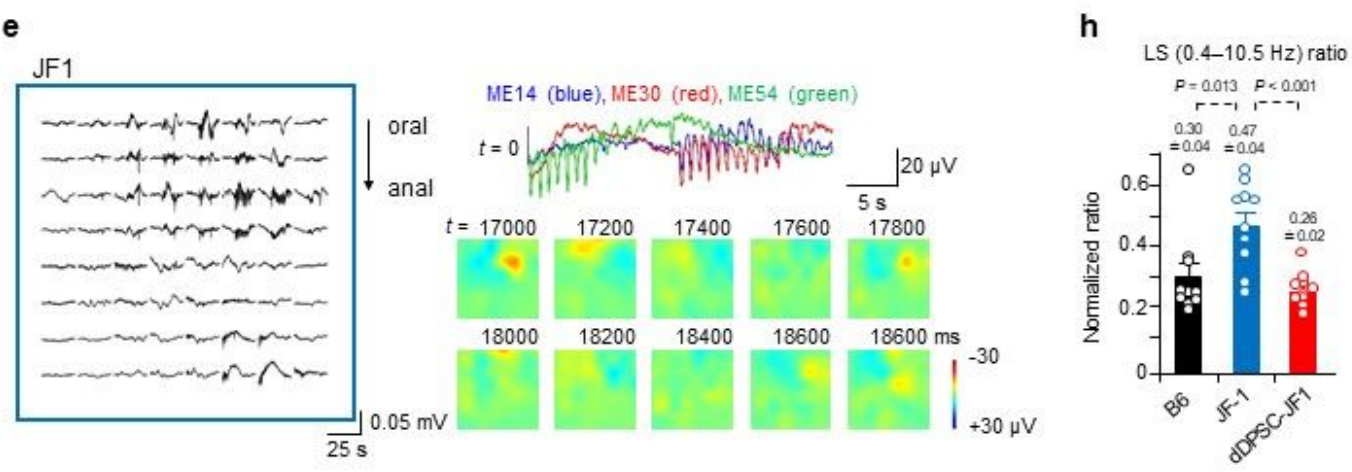

f

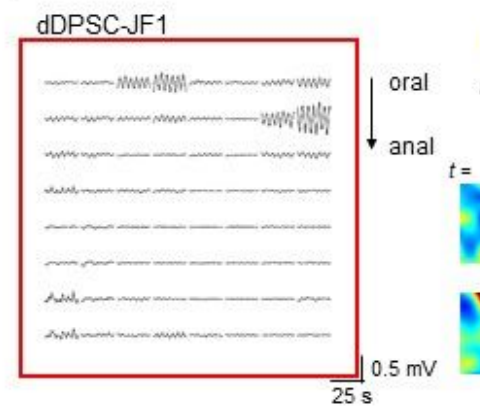

ME12 (blue), ME28 (red), ME44 (green)

$t=0$ fof A \& W A A $\frac{5 \mathrm{~s}}{50 \mu \mathrm{V}}$
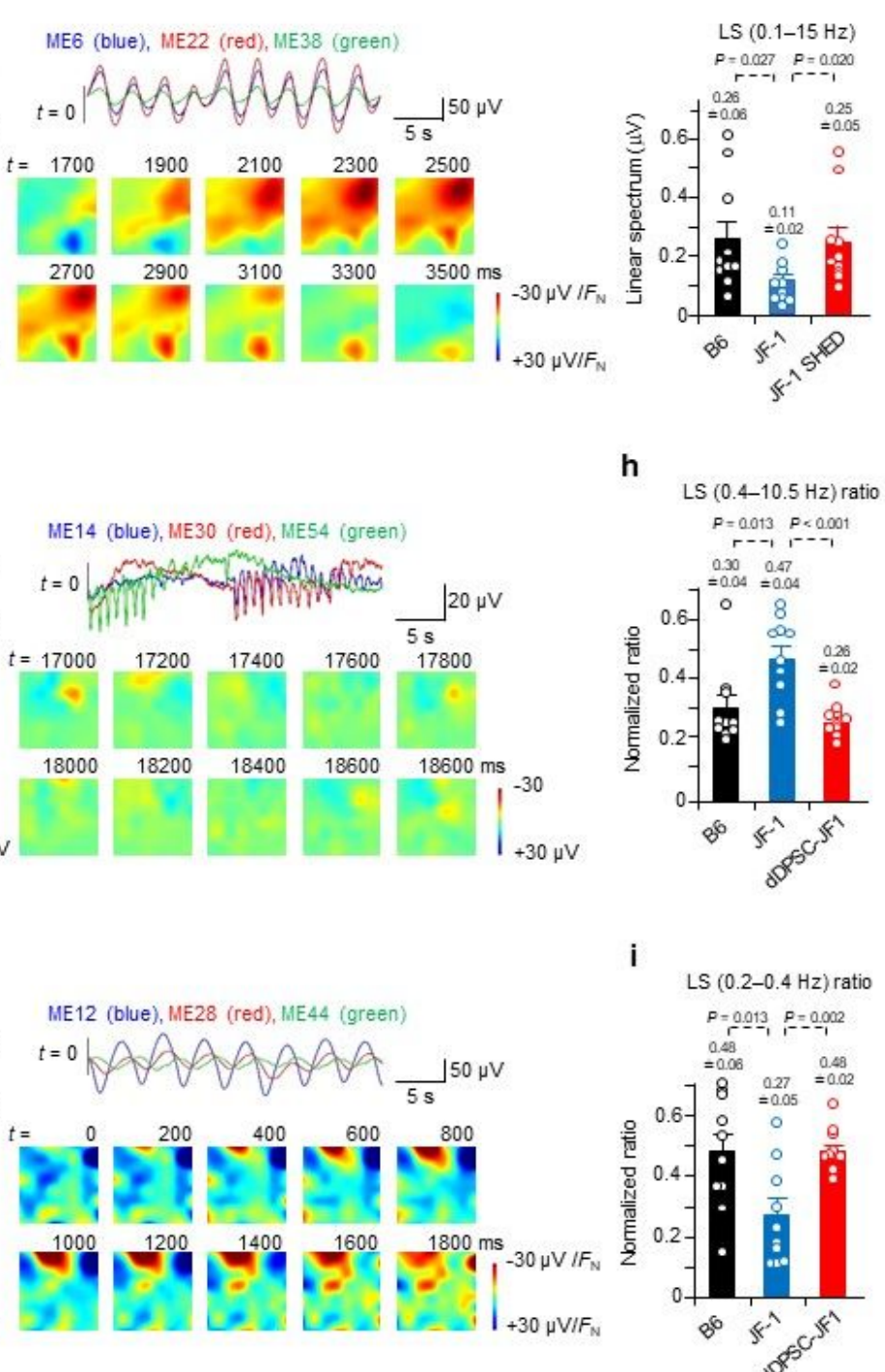

h

i

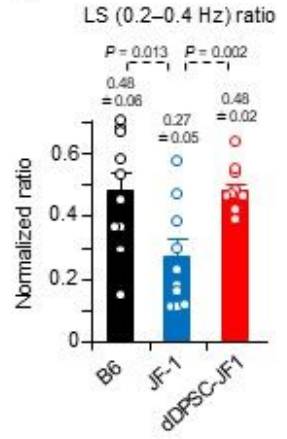

Figure 3 
Effects of dDPSCs on colonic function. a, Spontaneous contractions recorded from proximal colon muscle strips. b,c, Slow wave frequency (mean $\pm S E M, n=5$ ) and time-tension integral (area under the curve) for the spontaneous contractions (mean \pm SEM; B6, $n=6$; JF1, $n=14$; dDPSC-JF1, $n=7$ ). $d-f$, Electrical potentials recorded from the proximal colon with an $8 \times 8 \mathrm{MEA}$ and the corresponding reconstructed potential maps. $g-i$, Characteristics of the electrical potentials recorded with the $8 \times 8$ MEA: potential magnitude, proportion of high-frequency $(0.4-10.5 \mathrm{~Hz})$ potentials, and proportion of lowfrequency $(0.2-0.4 \mathrm{~Hz})$ potentials (mean $\pm S E M, n=3)$. a, $d$-f: Representative of $\geq 2$ independent experiments. 
a

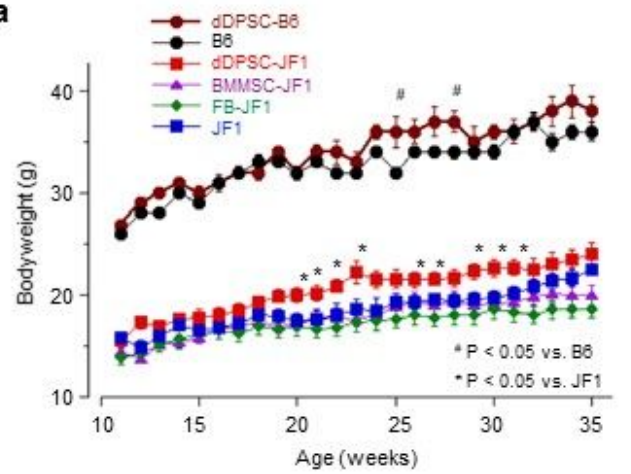

b

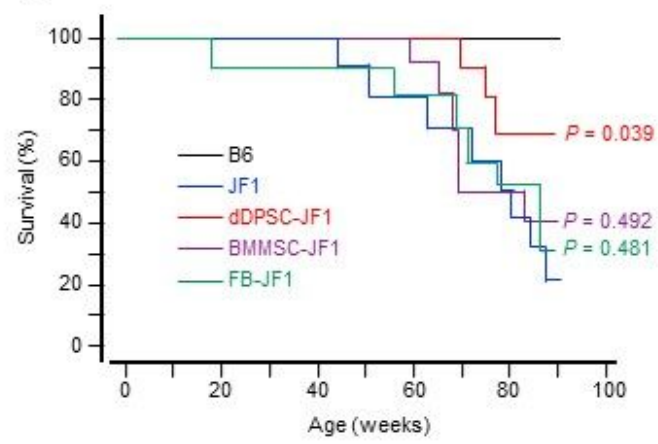

C
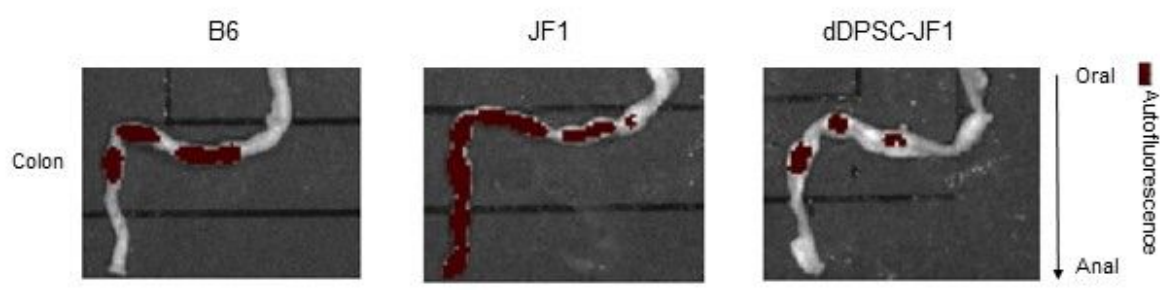

d

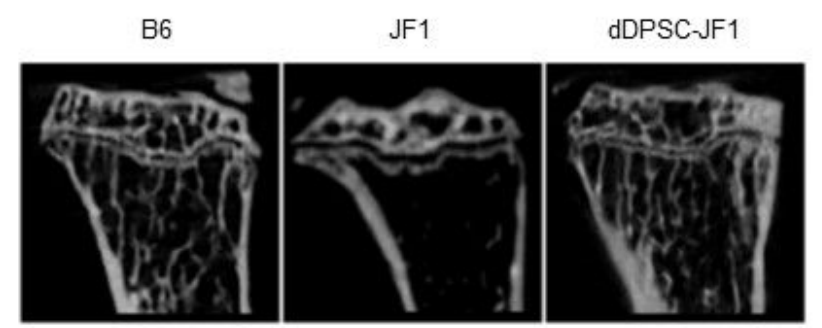

e

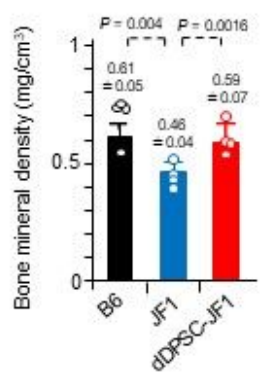

f

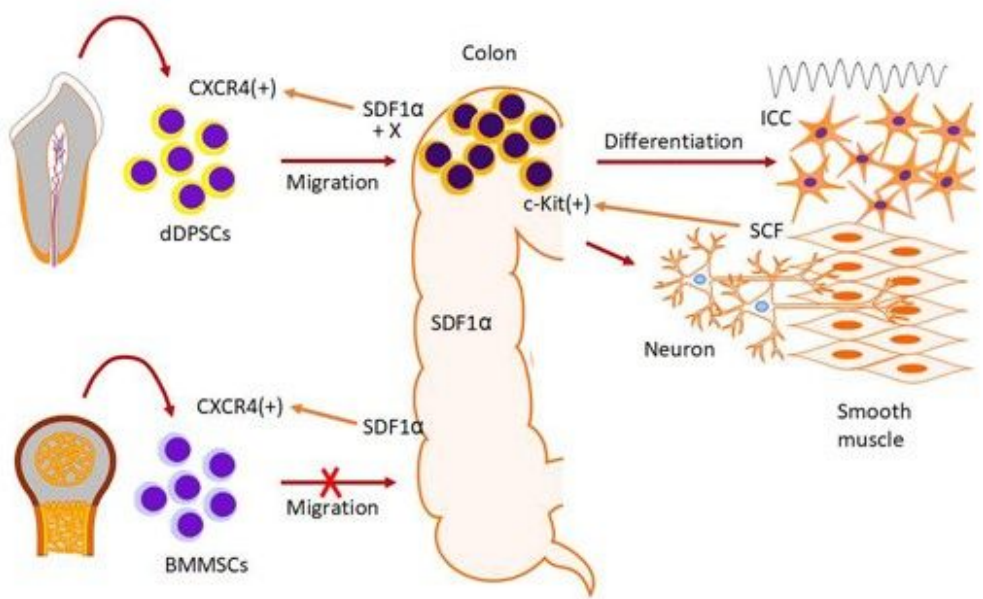

\section{Figure 4}

Therapeutic effects of dDPSCs. a, Mouse bodyweight changes after the transplantation of stem cells into JF1 (mean $\pm S E M, n=10)$ and B6 (mean $\pm S E M, n=5)$ mice. b, Kaplan-Meier survival curves $(n=10)$. Comparisons (vs. JF1 mice) were made using the log-rank test. c, Autofluorescence of the colonic contents after a 24 -hour fast. Representative of $\geq 2$ independent experiments. d, Micro-CT of the tibia. e, 
Tibial mineral density (mean $\pm S E M, n=4$ ). $f$, Schematic diagram showing the possible mechanisms underlying dDPSC migration, differentiation and beneficial effects on colonic motility in JF1 mice.

\section{Supplementary Files}

This is a list of supplementary files associated with this preprint. Click to download.

- FigS1.jpg

- FigS2.jpg

- FigS3.jpg

- FigS4.jpg

- FigS520210719.jpg

- FigS6.jpg

- FigS7.jpg

- FigS8.jpg

- SupplementaryTables.pdf 